\section{Taking the easy way out?}

Immature dendritic cells (DCs) in our immune system continuously sample the environment for rogue antigens, ready to alert the body to any foreign invaders. These antigens accumulate in the lysosomes of DCs together with antigen-presenting major histocompatability complex class-II (MHC-II) molecules. During DC maturation, antigen-derived peptides are then loaded onto the MHC-II molecules and both are rapidly redistributed to the cell surface for recognition by $\mathrm{T}$ cells. Through the elegant use of live cell imaging, Mellman and colleagues (Nature 418, DOI: 10.1038/nature01006 (2002)) have now identified a novel retrograde pathway by which these complexes exit lysosomes, and thus they explain how these complexes are rapidly retrieved from what is classically thought to be a 'dead end' for endocytosed material.

To study retrieval from lysosomes in living cells, Mellman and colleagues made an MHC-II-green fluorescent protein (GFP) fusion protein (shown in green in the figure). MHC-II-GFP recapitulated the dynamic localization pattern of endogenous MHC-II, residing in the lysosomes of immature DCs (left, shown in red) and then rapidly redistributing to the plasma membrane after maturation (right).

So how do the MHC II complexes escape the lysosomes and move to the cell surface? Previous work has shown that activation of DC-like cells (J. Cell Biol. 155, 53-63 (2001)) coincides with a marked tubulation of lysosomal membranes. Thus, it was speculated that these tubules might aid transport to the plasma membrane. Using MHC-II-GFP, Mellman and colleagues imaged DCs during maturation and observed that the lysosomal structures underwent marked morphological changes, forming long tubules. As they looked in more detail, they noticed that these tubules were transient, separating off and moving towards the cell surface. Interestingly, most of the lysosomal content was excluded from the

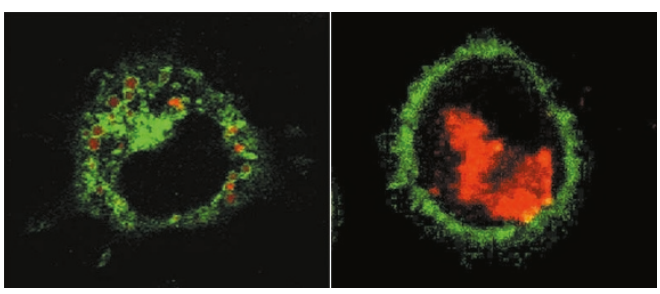

tubules, suggesting that the movement of MHC II molecules out of lysosomes is selective and regulated.

To test whether these tubules do actually mediate transport to the cell surface, they used total internal reflection microscopy (TIRFM), one of the few techniques that allows visualization of fusion events at the plasma membrane. As suspected, the MHC II-GFPexpressing vesicles/tubules underwent fusion with the plasma membrane. To prove that these tubules were indeed derived from lysosomes, the authors confirmed that they also contained an antigen that is known to associate with MHC II molecules in lysosomes only.

Clearly, this pathway must be tightly regulated so that tubulation begins precisely when cells need to begin presenting antigen to $\mathrm{T}$ cells. So what are the underlying cytoskeletal dynamics that drive this process and how is it regulated? Interestingly, the prime candidates - microtubules - have previously been shown not to be required for the recruitment of MHC from lysosomes. On another point, what is the sorting mechanism in lysosomes that allows transport of these complexes, but leaves behind the bulk of lysosomal content? And, importantly, are there similar escape pathways from lysosomes in other cell types or is this unique to DCs? No doubt, it will not be long before we are given the first glimpse of answers to these questions.

ALISON SCHULDT

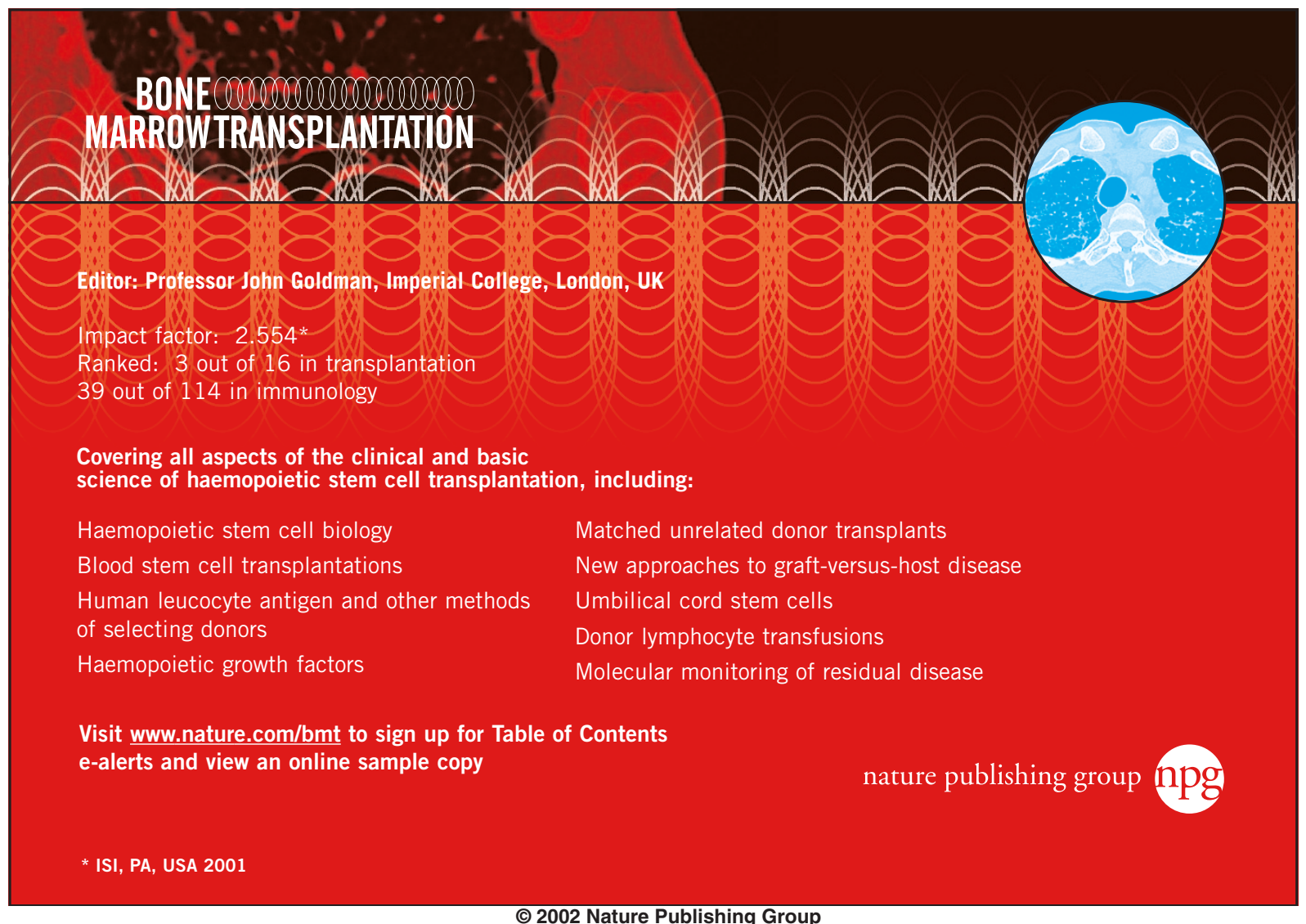

